\title{
Reply to comment by Vallée et al. on "Earthquake-induced prompt gravity signals identified in dense array data in Japan"
}

\author{
Masaya Kimura ${ }^{*}$ (D), Nobuki Kame ${ }^{1}$, Shingo Watada ${ }^{1}$, Makiko Ohtani ${ }^{1}$, Akito Araya ${ }^{1}$, Yuichi Imanishi',
} Masaki Ando ${ }^{2}$ and Takashi Kunugi ${ }^{3}$

\begin{abstract}
Density perturbations accompanying seismic waves are expected to generate prompt gravity perturbations preceding the arrival of P-waves. Vallée et al. (Science 358:1164-1168, 2017, https://doi.org/10.1126/science.aao0746) reported the detection of such pre-P-wave signals in broadband seismograms during the 2011 Tohoku-oki earthquake. Kimura et al. (Earth Planets Space 71:27, 2019, https://doi.org/10.1186/s40623-019-1006-x) considered that their detection involved some uncertain points, including a concern regarding their signal processing procedure. Specifically, to remove the instrumental response, Vallée et al. (2017) applied acausal deconvolution to the seismograms truncated at the P-wave arrivals. Generally, acausal deconvolution produces artifacts at the edge of the time window. However, they did not present quantitative assessment whether the detected signals were artifacts due to the signal processing. To avoid this concern, Kimura et al. (2019) employed another procedure that eliminated acausal processes, resulting in the detection of a pre-P-wave signal with a statistical significance of $7 \sigma$ in stacked broadband seismograms. Subsequently, Vallée et al. (Earth Planets Space 71:51, 2019, https://doi.org/10.1186/s40623-019-1030-x) commented that the procedure employed by Kimura et al. (2019) for the signal detection was inappropriate because it dismissed the low-frequency components of data. Although we admit the loss of low-frequency components in the data in Kimura et al. (2019), Vallée et al. (2019) have not yet provided a full account of the validity of their own procedure. Here, we assessed the validity of the procedure employed by Vallée et al. (2017) by quantitatively evaluating the magnitude of the acausal artifacts. First, we investigated how the input acceleration waveform, having an ideal signal-like shape, was distorted by their procedure. Their acausal deconvolution indeed generated a large-amplitude terminal artifact; however, it was removed by the causal band-pass filtering performed after the deconvolution and consequently became negligible. Next, we constrained the maximum amplitude of the artifact due to the noise in a seismogram and showed that it was sufficiently small compared to the reported signal amplitudes. These results suggest that the signal waveforms seen after their procedure were not artifacts but were representing the input acceleration with sufficient accuracy. Namely, their procedure well functions as a detection method for pre-P-wave signals. In the context of this validation, we replied to the comments of Vallée et al. (2019).
\end{abstract}

Keywords: Prompt elastogravity signals, Deconvolution, Acausal signal processing, 2011 Tohoku-oki earthquake

This comment refers to the article available at https://doi.org/10.1186/s4062

3-019-1006-x.

*Correspondence: mkimura@eri.u-tokyo.ac.jp

1 Earthquake Research Institute, The University of Tokyo, Yayoi, Bunkyo-ku,

Tokyo 113-0032, Japan

Full list of author information is available at the end of the article 


\section{Introduction}

We express gratitude to Vallée et al. (2019) (hereafter referred to as V19) for their valuable comments on our recent paper (Kimura et al. 2019, hereafter referred to as K19). Their comments provided us an opportunity to discuss interesting points, especially about signal processing methods. We summarize the controversial points discussed in K19 and V19.

Compressional seismic waves radiating from an earthquake carry density perturbations, which generate transient perturbations in the gravity field. Since variations in gravity propagate at the speed of light, observers are able to detect them prior to the arrival of the direct P-waves. Vallée et al. (2017) (hereafter referred to as V17) reported the detection of such pre-P-wave signals, which they called prompt elastogravity signals, in the records of broadband seismometers during the $2011 \mathrm{Mw} 9.1$ Tohoku-oki earthquake (Fig. 1 in V17). The signals were well simulated by a combination of the pre-P-wave gravity variation and ground motion induced by the gravity variation in a half-space model (Figs. 2, 3 in V17). However, K19 considered that there was a possibility that the reported signals were not real signals from the earthquake but artifacts of their signal processing, local noise, or simple background noise because of the following problems:

\section{A1. Acausal signal processing}

The signal processing procedure in V17 (hereafter referred to as Proc. V) was as follows: (i) the seismograms were truncated at the $\mathrm{P}$-wave arrival time, $t_{\mathrm{P}}$; (ii) the mean value was subtracted; (iii) the sensor response was deconvolved using the "transfer" command of the Seismic Analysis Code (SAC, Goldstein and Snoke 2005); and (iv) a 0.002$0.03-\mathrm{Hz}$ band-pass filter was applied. Step (iii) was acausal because it was calculated in the frequency domain, which generally introduces a ringing artifact at the end of the time series, i.e., prior to $t_{\mathrm{P}}$ (e.g. Havskov and Alguacil 2016; Press et al. 1992; Stein and Wysession 2003). Preceding the usage of Proc. V, it should have been confirmed that the acausal effect was negligible and that Proc. V could restore the input acceleration waveform with sufficient accuracy; however, such a confirmation was not fully accomplished in V17. Therefore, it is still unclear whether the reported signals were real or only artifacts. Additionally, the comparison with the theoretical waveforms was also unconvincing because the restoration of the input acceleration waveforms was not guaranteed. Note that step (iv) was causal; V17 used 0.002-Hz two-pole high-pass and $0.03-\mathrm{Hz}$ six-pole low-pass causal Butterworth filters.

A2. Limited data use

V17 showed the data recorded at only 11 observation stations and did not mention nearly 200 other stations in East Asia. Although V17 claimed that the 11 stations were selected from low-noise-level stations avoiding redundancy, they did not explain how many stations satisfied their noise-level criterion and whether the data from the other stations contained similar trends prior to $t_{\mathrm{p}}$. Therefore, the influence of the local noise could not be denied. Generally, utilizing several stations at similar locations is preferable because it can be used for discriminating the common signal from the local noise.

A3. Unclear significance of the signals

The statistical significance of the reported signals was not discussed and, therefore, the rarity of the signals was not clear. Although V17 newly developed a model that seemed to well explain the 11 signal waveforms, the model had not been established and did not have evidence that it could accurately predict the prompt elastogravity signals. Thus, statistical evidence was necessary for the claim of the signal detection.

K19 resolved these problems and showed a statistically significant signal preceding the P-waves originating from the 2011 Tohoku-oki event as follows:

B1. While Proc. V suffered from the acausal problem in the deconvolution process, K19 avoided this and replaced the process with the division of the output of the seismometer by the sensor sensitivity coefficient. This coefficient was defined as the velocityto-count factor within the frequency band where the instrument response was flat. This process was causal. Moreover, K19 used the same causal bandpass filter as in Proc. V. Consequently, the entire process of the signal processing procedure of K19 (hereafter referred to as Proc. K) was causal, which enabled K19 to discuss the presence of the pre-Pwave signals without any influence of the post-Pwave information after time $t_{\mathrm{P}}$. See Appendix 2 of K19 for reference.

B2. K19 referred to all the data that they used: two superconducting gravimeters, 71 broadband seismometers, and 706 tiltmeters in Japan. Although most of the single-channel records did not show any signals (Fig. 6, S2 in K19), a clear signal was discovered in the stacked broadband seismometer data (Fig. 7 in K19). 
B3. It was found that the statistical significance of the signal in the stacked trace was $7 \sigma$ with respect to the background noise level.

Following the above analyses, K19 discussed the signal detection and model of V17 as follows: (i) the signals reported by V17 might have been noise or artifacts due to their acausal signal processing because most nearby stations that were not used in V17 did not show any signal following Proc. K, and (ii) for the 2011 event, the model of V17 seemed to overestimate the signal amplitude; the amplitude of the stacked trace of $\mathrm{K} 19$ was $0.25 \mathrm{~nm} / \mathrm{s}^{2}$, whereas the typical amplitude of the model prediction of V17 was $1 \mathrm{~nm} / \mathrm{s}^{2}$.

Then, V19 commented on K19 as follows:

C1. Proc. K was flawed. Proc. K involved an incomplete correction of the instrument response that dismissed the low-frequency components of the broadband seismometer data, particularly of the STS-2 sensor data (Fig. 1 in V19). Consequently, K19 failed to confirm the signal detection by V17.

C2. K19 did not consider the station quality. K19 erroneously discarded the signals at high-quality stations on the basis of their negative results at the neighboring noisy stations.

C3. The claims of originality by K19 were invalid because they were based on inappropriate data processing. The detection significance of the stacked waveform of K19 was lower than even that of only one of the individual signals shown in V17.

C4. Stacking the same data as K19 following Proc. V resulted in a signal with the same amplitude as that predicted by the model of V17 and with a much higher significance than K19.

These comments were based on the assumption that Proc. V was appropriate for the detection of prompt elastogravity signals. However, we think that this supposition has not yet been confirmed even by V19, despite the problem presentation by K19. Although V19 recognized the existence of the acausal effect, they claimed without any quantitative evidence that the effect became problematic only if the deconvolution was applied to a time series containing an undesirable subsequent signal (which is thought to mean a direct P-wave). Moreover, they claimed that an arbitrary choice of the time window did not affect the acceleration waveform obtained by Proc. V, i.e., Proc. V provided the same acceleration signals in the frequency range of $0.002-0.03 \mathrm{~Hz}$ regardless of the choice of the time window, as long as it was sufficiently long and the P-wave was not included. If it is shown that any obtained waveform was always independent of the end limit of the time window, then it indicates that Proc. V is causal and has no issues. However, they tested the variation in the end limit only for three cases: $t_{\mathrm{P}}, t_{\mathrm{P}}-5 \mathrm{~s}$, and $t_{\mathrm{P}}-10 \mathrm{~s}$, which seem to be far from the arbitrary choice considering that the signal durations were of the order of $100 \mathrm{~s}$ (Fig. 2 in V19). Moreover, no quantitative evaluation was performed about the influence of the time window on the obtained waveforms. More general verification or explanation is thought to be necessary. In addition, they used the data of only two stations for the test. Therefore, we continue to be concerned about applying Proc. V to any other seismogram.

Here, before replying to the comments by V19, we solve the still existing question about the signal processing: "does the waveform obtained by applying Proc. V represent the input acceleration?" ("Verification of the signal processing procedure employed by Vallée et al. (2017)" section). Initially, we show that Proc. V can well restore the ideal input acceleration waveform for the case of a transient acceleration input simulating a prompt elastogravity signal. The acausal deconvolution process generated a large-amplitude terminal artifact; however, it was effectively removed by the phase delay introduced by applying the causal band-pass filter. Therefore, the waveform obtained by applying Proc. V was nearly equal to the band-pass-filtered input waveform with a small relative error. Furthermore, we evaluate the upper limit of the terminal artifact in the presence of background noise and show that it is smaller than or comparable to the reported signal amplitudes. These results indicate that the acausal effect is practically negligible and Proc. V can reasonably well restore the input acceleration waveforms. Thus, we can regard Proc. V as an acceptable method to explore prompt elastogravity signals. Finally, we reply to the comments of V19 ("Reply to comments" section).

\section{Verification of the signal processing procedure employed by Vallée et al. (2017)}

First, we consider the case where the input acceleration is an ideal signal waveform and see the results of each process of Proc. V. Fig. 1a is an example of the simulated waveform of a prompt elastogravity signal. It increases proportionally to $t^{5}$ within certain time limits (here between $t=0$ and $150 \mathrm{~s}$ ) assuming that the initial increase of the moment rate is proportional to $t^{2}$, and no noise is contained. For this acceleration input, the expected output of a broadband seismometer is displayed in Fig. 1b. Then, we manually truncated the output at a certain time (here $t=100 \mathrm{~s}$ when the input acceleration reaches its amplitude of $1 \mathrm{~nm} / \mathrm{s}^{2}$ ) and deconvolved the sensor response as in step (i) and (ii) of Proc. V (Fig. 1c). The deconvolved output had a ringing artifact immediately before the end limit, and the amplitude of the 

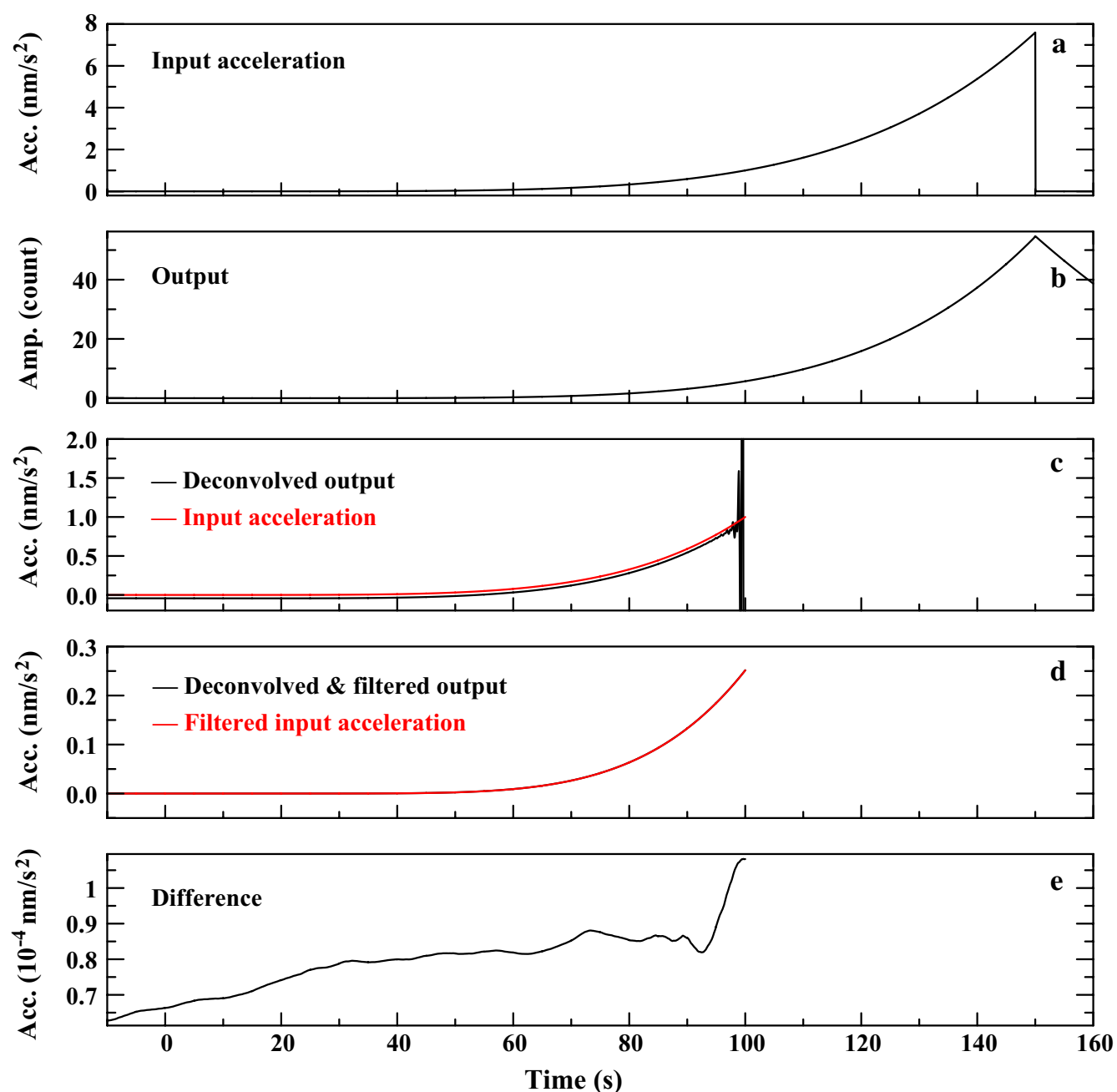

Fig. 1 Step-by-step results of the signal processing procedure employed by Vallée et al. (2017). "Acc." and "Amp." are the abbreviations of "Acceleration" and "Amplitude", respectively. a Input acceleration waveform, which increases in proportion to $t^{5}$ for $t=0-150 \mathrm{~s}$ and is zero before and after the time range. The sampling rate, number of points, and time window are $20 \mathrm{~Hz}, 72,000$, and $t=-1800-1800 \mathrm{~s}$, respectively. $\mathbf{b}$ Expected output waveform from the broadband seismometer installed at FUK (Fukue). Here, the instrument response of the seismometer was convolved in the frequency domain using the "transfer" command of SAC. c Truncated at $t=100 \mathrm{~s}$ and deconvolved output waveform (black) as well as truncated input acceleration waveform (red). Here, the deconvolution was performed using the "transfer" command, as before. The offset of the black curve is thought to be due to the zero response at zero frequency. $\mathbf{d}$ Truncated, deconvolved, and 0.002-0.03-Hz band-pass-filtered output waveform (black) as well as truncated and band-pass-filtered input acceleration waveform (red). e Difference between them, i.e., black curve minus red curve in $\mathbf{d}$

artifact was 30 times larger than that of the original input amplitude. Next, we applied the same causal band-pass filter as in step (iii) of Proc. V to both the deconvolved output and original input (Fig. 1d). The ringing artifact observed in the deconvolved output disappeared following the filtering, and the deconvolved and filtered output well agreed with the filtered input. Their difference was less than $0.1 \%$ compared to their amplitudes (Fig. 1e). This result exhibits that in this ideal case, the waveform obtained by applying Proc. V, i.e., deconvolved and filtered output, well represents the input acceleration in the frequency band. We conducted similar tests using simulation waveforms that increase in proportion to $t^{1}, t^{2}, t^{3}$, and $t^{4}$. As before, the deconvolved and filtered output agreed with the filtered input with an error less than $0.2 \%$ (Additional file 1: Figs. S1-S4). Additional file 1: Figs. S5 and S6 show the results for the case of different signal durations. The input acceleration was also restored.

The disappearance of the ringing artifact following the band-pass filtering is attributed to the phase delay of the employed filters. The causal low-pass and highpass Butterworth filters used in Proc. V are accompanied by frequency-dependent phase delays. The combined 
phase-delay time of the two filters is more than $20 \mathrm{~s}$ in their pass band of $0.002-0.03 \mathrm{~Hz}$ (Fig. 2). However, the artifacts were limited within $3 \mathrm{~s}$ prior to the end of the time window (Fig. 1c). We can interpret that this time delay pushed out the artifact from the time window, and thus the artifact seemed to disappear.

Next, we consider the case where the seismograms contain noise and evaluate the upper limit of the terminal artifacts due to the noise. For this purpose, we first focus on the origin of the ringing artifact, as shown in Fig. 1c. Because each process of Proc. $\mathrm{V}$ is linear, the deconvolution result can be written as a superposition of the results for the impulse time series as follows:

$$
\begin{aligned}
c & =f(\boldsymbol{b}) \\
& =f\left(\sum_{j=1}^{N} b_{j} \boldsymbol{\delta}^{j}\right) \\
& =\sum_{j=1}^{N} b_{j} f\left(\boldsymbol{\delta}^{j}\right) \\
& =\sum_{j=1}^{N} b_{j} \boldsymbol{h}^{j} .
\end{aligned}
$$

Here, $N$ is the data length, $\boldsymbol{b}=\left(b_{i}\right)_{i=1 \sim N}$ denotes the extracted output of the seismometer, $\boldsymbol{c}=\left(c_{i}\right)_{i=1 \sim N}$ is the deconvolved output, $f$ is step (ii) and (iii) of Proc. V, i.e., subtracting the mean value and deconvolving the sensor response, $\boldsymbol{\delta}^{j}=\left(\delta_{i}^{j}\right)_{i=1 \sim N}$ is a delta-like time series, i.e.,

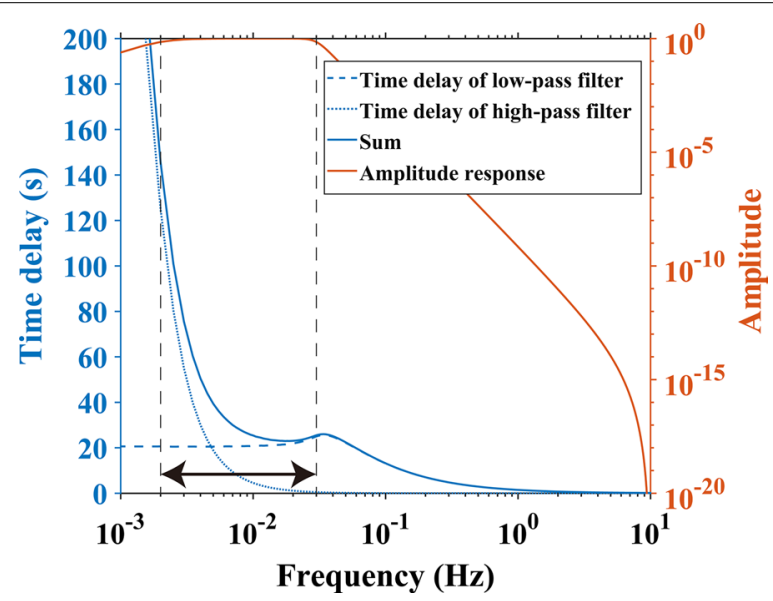

Fig. 2 Time delay of the six-pole $0.03-\mathrm{Hz}$ low-pass Butterworth filter (dashed blue line), time delay of the two-pole $0.002-\mathrm{Hz}$ high-pass Butterworth filter (dotted blue line), sum of the above two time delays (solid blue line), and amplitude response of the two filters (solid red line). These Butterworth filters were used in V17 and K19. Vertical dashed lines and horizontal arrows indicate the pass band of $0.002-0.03 \mathrm{~Hz}$ $\delta_{i}^{j}=1$ only when $i=j$ and otherwise 0 , and $\boldsymbol{h}^{j}=\left(h_{i}^{j}\right)_{i=1 \sim N}$ is the impulse response of $f$ for $\boldsymbol{\delta}^{j}$. While $\boldsymbol{h}^{j}$ was rich in a short-period oscillation component as shown in Fig. 3a, the deconvolved output became smooth except the terminal portion (Fig. 1c). This is because the short-period component of $\boldsymbol{h}^{j}$ was cancelled by multiplying $\boldsymbol{h}^{j}$ by a smooth waveform like Fig. $1 \mathrm{~b}$ and taking the summation over $j$. Conversely, we can infer that the ringing artifact emerges at the location where the short-period component is not cancelled. Here, we note that the "transfer" command of SAC pads the data with zeroes to the next power of two $\left(2^{k}, k \in \mathbb{Z}^{+}\right)$to use the fast Fourier transform (Goldstein and Snoke 2005). Namely, extracting an $N$-long time series $(i=1 \sim N)$ corresponds to extracting a $2^{k}$-long time series $\left(i=1 \sim 2^{k}\right)$ and replacing the time series of $i=N+1 \sim 2^{k}$ with zeroes. Therefore, the terminal ringing artifact as seen in Fig. 1c is considered to be generated by the absence of the contribution of the following data points: $\sum_{j=N+1}^{2^{k}} b_{j} \boldsymbol{h}^{j}$, which will cancel the artifact if the output is extracted with a length of $2^{k}$ and not replaced with zeroes. The artifact owing to the truncation and deconvolution can be evaluated as $-\sum_{j=N+1}^{2^{k}} b_{j} \boldsymbol{h}^{j}$. In addition, the duration of the terminal artifact is expected to be equal to the duration of the impulse response $\boldsymbol{h}^{j}$ prior to the input impulse (Figs. 1c, 3a).

This formulation can be directly applicable to estimate the terminal artifact generated by applying entire Proc. V to a seismogram containing noise. In many cases, the dominant noise in the broadband seismograms is microseism noise, and interrupting such a noise waveform at the end limit of the time window can also become a source of an artifact. A possible artifact, $\boldsymbol{s}=\left(s_{i}\right)_{i=1 \sim N}$, generated by the entire Proc. V is evaluated as $s=-\sum_{j=N+1}^{2^{2}} b_{j} \boldsymbol{g}^{j}$, where $\boldsymbol{g}^{j}=\left(g_{i}^{j}\right)_{i=1 \sim N}$ is the impulse response of $f^{\prime}$ for $\delta^{j}$ and $f^{\prime}$ is step (ii), (iii), and (iv) of Proc. V, i.e., subtracting the mean value, deconvolving the sensor response, and applying the causal low-pass and high-pass filters. Figure $3 \mathrm{~b}$ shows an example of $\boldsymbol{g}^{j}$. In the presence of noise with a maximum amplitude $A$, the maximum value of the amplitude of the artifact at the end of the obtained time series, $\left|s_{N}\right|$, is evaluated as follows:

$$
\begin{aligned}
\left|s_{N}\right|=\mid & -\sum_{j=N+1}^{2^{k}} b_{j} g_{N}^{j} \mid \\
& \leq \sum_{j=N+1}^{2^{k}}\left|b_{j} g_{N}^{j}\right| \\
& \leq A \sum_{j=N+1}^{2^{k}}\left|g_{N}^{j}\right| .
\end{aligned}
$$



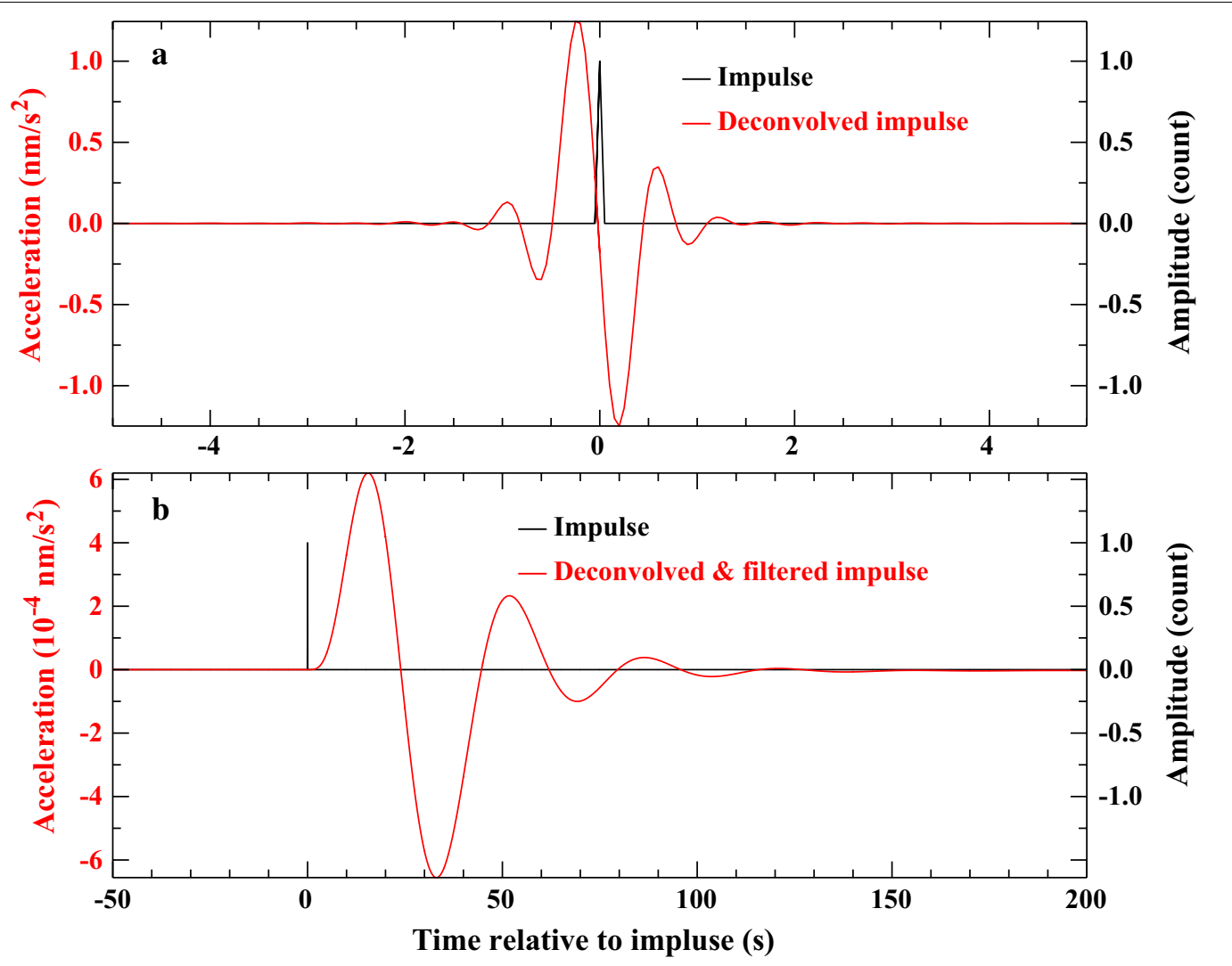

Fig. 3 An example of the impulse response of Proc. V. a Impulsive time series $\boldsymbol{\delta}^{j}=\left(\delta_{i}^{j}\right)_{i=1 \sim N}\left(\right.$ black) and deconvolved impulse $\boldsymbol{h}^{j}=\left(h_{i}^{j}\right)_{i=1 \sim N}$ (red). Here, the instrument response of the FUK broadband seismometer was deconvolved using the "transfer" command of SAC. $\mathbf{b}$ Impulsive time series $\boldsymbol{\delta}^{j}=\left(\delta_{i}^{j}\right)_{i=1 \sim N}$ (black) and deconvolved and 0.002-0.03-Hz band-pass-filtered impulse $\boldsymbol{g}^{j}=\left(g_{i}^{j}\right)_{i=1 \sim N}($ red $)$

For example, the noise level of the Fukue (FUK) STS-1 broadband seismometer data was approximately $2 \times 10^{2}$ counts at the time of the 2011 event occurrence, and $\sum_{j=N+1}^{2^{k}}\left|g_{N}^{j}\right|=6 \times 10^{-4} \mathrm{~nm} / \mathrm{s}^{2}$ for a sufficiently long data length $N$ (here we set $N=108000$, i.e., 90 -min data). Thus, $\left|s_{N}\right|$ was constrained as $\left|s_{N}\right|<0.1 \mathrm{~nm} / \mathrm{s}^{2}$. Because the signal amplitude that V17 reported at the station was $1 \mathrm{~nm} / \mathrm{s}^{2}$, which is larger than the calculated maximum $\left|s_{N}\right|$, so the reported signal is not considered as an artifact. The noise level of the Matsushiro (MAJO) STS-2 seismometer data was of the order of $10^{4}$ counts, and $\sum_{j=N+1}^{2^{k}}\left|g_{N}^{j}\right|=8 \times 10^{-5} \mathrm{~nm} / \mathrm{s}^{2}$, so that $\left|s_{N}\right|$ was constrained as $\left|s_{N}\right|<0.8 \mathrm{~nm} / \mathrm{s}^{2}$. The reported signal amplitude was approximately $0.7 \mathrm{~nm} / \mathrm{s}^{2}$, which is comparable to the upper limit, but is also sufficiently large not to be considered as an artifact. This is because the upper limit is obtained when the extracted seismogram $b_{j}=A \operatorname{sign}\left(g_{N}^{j}\right)$ for all $j(>N)$ and is highly overestimated for actual noise data.

The above results show that Proc. V is a practically valid procedure for detecting prompt elastogravity signals. As we saw, the large-amplitude ringing artifacts due to the acausal deconvolution (Fig. 1c) disappeared following the application of the causal band-pass filter (Fig. 1d). The comparison of the waveform obtained by applying Proc. V and the filtered acceleration input (Fig. 1e) showed that Proc. V can acceptably restore the input acceleration from the output of the seismometer for monotonically increasing signal inputs without noise, mimicking the prompt elastogravity signals of the 2011 event. Because the obtained waveform closely reproduced the input acceleration, we can compare it to the theoretically predicted waveforms and discuss how a model can simulate prompt elastogravity signals. The causal band-pass filter played a crucial role in Proc. V: it removed the terminal artifacts to the outside of the time window through its phase delay and made Proc. $\mathrm{V}$ virtually causal as a whole. We constrained the upper limit of artifacts due to noise, and its amplitude was sufficiently small compared to the reported signal amplitudes. Therefore, the signals reported by V17 are not considered to be acausal artifacts from the truncation and deconvolution. To claim that the reported signals 
were real signals from the 2011 event, we need to reject a possibility of local noise as noted in Introduction.

\section{Reply to comments}

\section{Strength and weakness of Proc. K}

Proc. $\mathrm{K}$ was designed to ensure the perfect causality of the signal processing. Although we showed above that the influence of the acausal deconvolution in Proc. V is practically negligible following the application of the band-pass filter, this had not been confirmed at the time of writing K19. We think Proc. K was at that time the best method for clear signal detection, and it is inappropriate to consider Proc. $\mathrm{K}$ as a flawed method before verifying Proc. V. However, as V19 pointed out, Proc. K loses the signal amplitude in the lower frequencies because of its incomplete correction of the instrument response using a frequency-independent constant sensitivity. This may be the reason why K19 could not detect clear signals in most single-channel records. Now that the practical validity of Proc. V has been confirmed, we accept that Proc. V is more advantageous than Proc. $\mathrm{K}$ in terms of the signal observations at low frequencies. In addition, we acknowledge that the argument of K19 about the observations of V17 was inadequate. K19 argued that the reported signals of V17 were only noise or artifacts because most of the neighboring stations did not show any signal following Proc. K. This argument came from the limited data use in V17, who did not show the data of any neighboring station. However, now we realize that the argument was misleading because K19 compared the results of two different methods: Proc. V and K.

\section{Quality control of the observation stations}

V17 selected a very limited number of observation stations based on their background-noise criterion and did not utilize others, which does not seem to be the best way to search for a signal. Because of this very limited sampling, V17 could not deny the possibility that the signals at the 11 stations were mere local noise due to, e.g., a local variation of the atmospheric pressure. To make the signal detection more reliable, not only champion data but also others should be shown. Generally, any observed data can contribute to noise reduction unless it has an extremely small signal-to-noise ratio. Station stacking is an effective method to reduce the local noise. Indeed, by station stacking in K19, the signal significance has increased to $7 \sigma$, and V19 followed the stacking method of K19 and achieved a signal significance of $14 \sigma$.

\section{Originality of K19}

The originality of K19 is that they used a completely causal signal processing procedure and discovered a signal with a statistical significance of $7 \sigma$ in a stacked trace. Their approach is independent of that of V17, who employed a procedure which had not been fully validated at that time, used data at limited number of stations, and did not show a statistical significance of their signals. V19 examined the results of V17 and claimed that the waveform at the Mudanjiang (MDJ) station had a higher significance of $9 \sigma$ than that of K19. However, such a retrospective claim does not degrade the originality of K19. Moreover, as we mentioned in the "Quality control of the observation stations" section, the influence of the local noise cannot be denied.

\section{Validity of the model of V17}

We agree with the comment of V19 on the argument of K19 about the validity of the model of V17. K19 compared the results of Proc. $\mathrm{K}$ to those of the prediction of the model of V17. However, this direct comparison is incorrect because Proc. K reduced the low-frequency amplitude as pointed by V19. The argument of V19 that the difference in the signal amplitudes of V17 and K19 can be explained by the recovery of the low-frequency amplitude is reasonable.

\section{Discussion}

\section{Waveform distortion due to filtering}

The band-pass filtering reduced the signal amplitude to approximately one quarter (Fig. 1c, d). This reduction rate depends on the signal duration: it was 0.075 when the duration was $50 \mathrm{~s}$ and 0.34 for $200 \mathrm{~s}$ (Additional file 1: Figs. S5c, S5d, S6c, S6d). In the case of elastogravity signals, the signal duration is proportional to the hypocentral distance. Therefore, the filtered amplitudes vary with the hypocentral distance even for the same input signal amplitudes. Conversely, the consistent amplitude of approximately $1 \mathrm{~nm} / \mathrm{s}^{2}$ of the signals reported in V17 over a wide region of East Asia does not imply that the input signal amplitudes were the same. It instead suggests that larger amplitudes were input for the near stations than for the farther ones. This signal duration effect is attributed to the 20-s phase delay of the causal band-pass filter (Fig. 2). For the near stations, the 20-s time shift accounts for a large portion of the signal duration and, therefore, the signal amplitude is considerably reduced, whereas for the far stations, the time shift has a limited role.

The phase delay of the filter helps to select appropriate filters for less distorted signal waveforms. The phase 

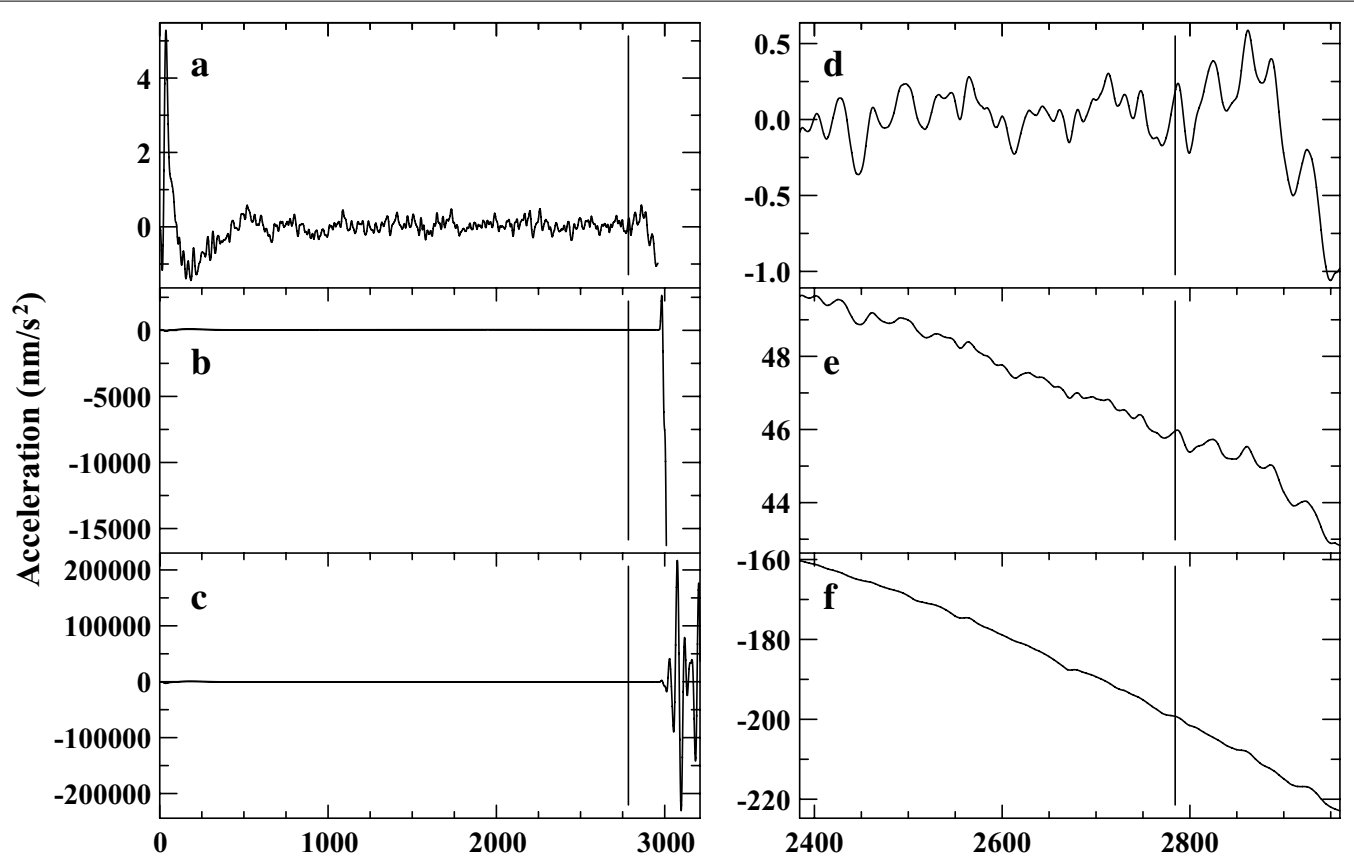

Time after 05:00 11 March 2011 (s)

Fig. 4 Acceleration waveforms at FUK at the time of the 2011 Tohoku-oki event following Proc. $V$ for the cases where a the output of the seismometer was truncated at the P-wave arrival time tp prior to the deconvolution, $\mathbf{b}$ the output was truncated at $t_{\mathrm{p}}+50 \mathrm{~s}$, and $\mathbf{c}$ the output was truncated at $t_{\mathrm{p}}+250 \mathrm{~s}$. d, e, and $\mathbf{f}$ are the enlarged views between $t_{\mathrm{eq}}-400 \mathrm{~s}$ (here $t_{\mathrm{eq}}$ denotes the event origin time) and $t_{\mathrm{p}}$ of $\mathbf{a}, \mathbf{b}$, and $\mathbf{c}$ respectively. The vertical lines indicate $t_{\text {eq. }}$. The output of the seismometer was not clipped before $t_{p}+250 \mathrm{~s}$

delay has to be sufficiently short to ensure that the signals are not pushed out of the time window. Simultaneously, it must be sufficiently long to remove the terminal artifact.

\section{Possible artifacts due to extremely large-amplitude seismic waves}

The validation of Proc. V showed that the band-pass filtering effectively removed the acausal artifacts and made Proc. V practically causal. Given this fact, it may be inferred that the seismograms do not need to be truncated at $t_{\mathrm{P}}$ before the deconvolution and can include the following large-amplitude seismic waves. Figure 4 shows the results of Proc. V applied to the seismograms truncated at $t_{\mathrm{P}}$ or containing the seismic waves from the 2011 event. In the latter cases (Fig. 4b, c, e, f), large-amplitude trends emerged and contaminated the trend clearly seen in Fig. $4 \mathrm{a}, \mathrm{d}$ before $t_{\mathrm{p}}$. Though we evaluated above that the artifact due to noise is negligible, it becomes apparent for a large-amplitude waveform (e.g., in the case of Fig. 4, the amplitude of the seismic waves was more than a thousand times larger than the noise level before deconvolution and band-pass filtering) as was already noted in V19. Therefore, we need to terminate the waveforms prior to the P-wave arrivals when using Proc. V.

\section{Conclusions}

V19 lead us to a deeper understanding of the signal processing methods in V17 and K19 than that we had previously. By confirming the restoration of the signal waveforms and evaluating the artifacts due to the truncated noise, we verified Proc. V as an appropriate method to search for prompt elastogravity signals. We found that Proc. V was practically valid owing to the characteristic phase-delay time of the band-pass filter, which could eliminate the ringing artifacts due to the acausal deconvolution. However, such an important point was not mentioned in V17 and V19. Research on prompt elastogravity signals thus far has been conducted by only a limited number of research groups, and many unclear points still exist. In this reply, we answered an unsolved fundamental problem about signal processing, which is critical for signal detection. We expect further progress in the field of prompt elastogravity signals. 


\section{Supplementary information}

Supplementary information accompanies this paper at https://doi. org/10.1186/s40623-019-1099-2.

Additional file 1. Fig. S1 Step-by-step results of the signal processing procedure employed by Vallée et al. (2017) for different input acceleration waveforms.

\section{Abbreviations}

FUK: Fukue; K19: Kimura et al. (2019); MAJO: Matsushiro; MDJ: Mudanjiang; SAC: Seismic Analysis Code; V17: Vallée et al. (2017);V19: Vallée et al. (2019).

\section{Acknowledgements}

We thank the Editor Masato Furuya and anonymous reviewers for their constructive comments that helped to improve the manuscript. We also thank Yasuo Ogawa, the Editor in Chief of Earth, Planets and Space, for his strong encouragement to submit our reply to the comment.

\section{Authors' contributions}

MK and SW performed the analysis. MK and NK wrote the manuscript. MK, NK, SW, and AA contributed to the planning. NK, SW, MO, AA, YI, MA, and TK contributed to the interpretation of the results and gave useful advice. All authors read and approved the final manuscript.

\section{Funding}

This research was supported by JSPS (KAKENHI JP15K13559, JP16K05532, JP18J21734) and MEXT via the Program for Leading Graduate Schools, the Earthquake and Volcano Hazards Observation and Research Program, and Quantum Leap Flagship Program (MEXT Q-LEAP, JPMXS01 18070351).

\section{Availability of data and materials}

The F-net data are available at NIED F-net server http://www.fnet.bosai.go.jp.

\section{Ethics approval and consent to participate}

Not applicable.

\section{Consent for publication}

Not applicable.

\section{Competing interests}

The authors declare that they have no competing interests.

\section{Author details}

${ }^{1}$ Earthquake Research Institute, The University of Tokyo, Yayoi, Bunkyo-ku, Tokyo 113-0032, Japan. ${ }^{2}$ Department of Physics, The University of Tokyo, Hongo, Bunkyo-ku, Tokyo 113-0033, Japan. ${ }^{3}$ National Research Institute for Earth Science and Disaster Resilience, Tennodai, Tsukuba 305-0006, Japan.

Received: 8 October 2019 Accepted: 27 October 2019

Published online: 14 November 2019

\section{References}

Goldstein P, Snoke A (2005) SAC availability for the IRIS community. IRIS DMC Newslett 7(1)

Havskov J, Alguacil G (2016) Instrumentation in earthquake seismology, 2nd edn. Springer, Dordrecht. https://doi.org/10.1007/978-3-319-21314-9

Kimura M, Kame N, Watada S, Ohtani M, Araya A, Imanishi Y, Ando M, Kunugi T (2019) Earthquake-induced prompt gravity signals identified in dense array data in Japan. Earth Planets Space 71:27. https://doi.org/10.1186/ s40623-019-1006-X

Press WH, Teukolsky SA, Vetterling WT, Flannery BP (1992) Numerical recipes in C: the art of scientific computing, 2nd edn. Cambridge Univ. Press, Cambridge

Stein S, Wysession M (2003) An introduction to seismology, earthquakes, and earth structure. Blackwell Publication, Malden

Vallée M, Ampuero JP, Juhel K, Bernard P, Montagner J-P, Barsuglia M (2017) Observations and modeling of the elastogravity signals preceding direct seismic waves. Science 358:1164-1168. https://doi.org/10.1126/science.aao0746

Vallée M, Ampuero JP, Juhel K, Bernard P, Montagner J-P, Barsuglia M et al (2019) Comment on "Earthquake-induced prompt gravity signals identified in dense array data in Japan" by Kimura et al. Earth Planets Space 71:51. https://doi.org/10.1186/s40623-019-1030-x

\section{Publisher's Note}

Springer Nature remains neutral with regard to jurisdictional claims in published maps and institutional affiliations.

\section{Submit your manuscript to a SpringerOpen ${ }^{\circ}$ journal and benefit from:}

- Convenient online submission

- Rigorous peer review

- Open access: articles freely available online

- High visibility within the field

- Retaining the copyright to your article

Submit your next manuscript at $\boldsymbol{\nabla}$ springeropen.com 